\title{
Comparative Study of Various Preparation Methods of Colloidal Silica
}

\author{
Hyung Mi Lim ${ }^{1 *}$, Jinho Lee ${ }^{1,2}$, Jeong-Hwan Jeong ${ }^{1,2}$, Seong-Geun $\mathrm{Oh}^{2}$, Seung-Ho Lee ${ }^{1}$ \\ ${ }^{1}$ Eco Composite Materials Center, KICET, Seoul, South Korea \\ ${ }^{2}$ Division of Materials Science Engineering, Hanyang University, Seoul, South Korea \\ E-mail:lim@kicet.re.kr \\ Received November 3, 2010; revised November 17, 2010; accepted November 22, 2010
}

\begin{abstract}
Colloidal silica can be prepared by various methods and starting materials including ion exchange of aqueous silicates, hydrolysis and condensation of silicon compounds, direct oxidation of silicon, and milling and peptization of silica powder. Various silica sols having particle sizes of 10-60 nm prepared by these methods and the preparation methods have been compared on the basis of their shape, size uniformity, sphericity, stability against $\mathrm{pH}$ variation, cation concentration, and price, etc. Silica sol prepared from tetraethoxysilane affords uniform size control and growth, and high purity, despite the relatively high costs. Silica sol prepared from liquid silicates affords relatively easy size and shape control; however, it is difficult to lower the alkali content to a level that is appropriate for carrying out semiconductor chemical mechanical polishing processes; in addition, the waste water treatment carried out for recovering the ion exchange resin gives rise environmental consideration. The properties of colloidal silica prepared from fumed silica powder by milling and dispersion depend on the starting silica source and it is relatively difficult to obtain monodispersed particles using this method. Colloidal silica prepared from silicon by direct oxidation has a monodispersed spherical shape and purity control with reasonable prices. It generates less waste water because it can be directly produced in relatively high concentrations. The cation fraction located in the particle relative to the free cation in the fluid is relatively lower in the silica sol prepared by the direct oxidation than others. A careful comparison of colloidal silica and the preparation methods may help in choosing the proper colloidal silica that is the most appropriate for the application being considered.
\end{abstract}

Keywords: Colloidal Silica, Comparison, Preparation Methods, Shape, Cation Location

\section{Introduction}

Colloidal silica is a stable dispersion of solid silica particles. It has found various applications such as investment casting, semiconductor wafer polishing, coating, and textiles, and it has also been used as an inorganic binder, a nano-size filler, and as a catalyst precursor [1,2]. Colloidal silica can be prepared by various methods and starting materials, as listed in Table 1 [2]. These methods include ion exchange [3-5], neutralization or electrodialysis of aqueous silicates, hydrolysis and condensation of silane [6], peptization or milling of silica gel or powder [7], and direct oxidation of silicon [8-10].

Silicon is the second most abundant element on earth, and it commonly exists in the form of silica ore. It is difficult to ensure high purity and size control of silica; the milling of naturally occurring silica ore results in even greater difficulties in the shape control of silica. The size and shape of nanosized silica particles in colloidal silica can be well controlled by synthesizing it from silica ore using various synthetic routes, as summarized in Figure 1. There are four typical routes to prepare colloidal silica from natural ore. The first among them is the preparation of colloidal silica from liquid sodium silicate by the ion exchange process. The sodium silicate is obtained from naturally available silica by melting the silica ore in the presence of alkali flux; subsequently, it is dissolved by heating under pressure to produce liquid sodium silicate, which is commonly known as water glass. The liquid sodium silicate has high viscosity, and therefore, it is diluted to a concentration of 3-5 wt\%. Next, it is passed through an ion-exchange resin, and then fed into the al- 


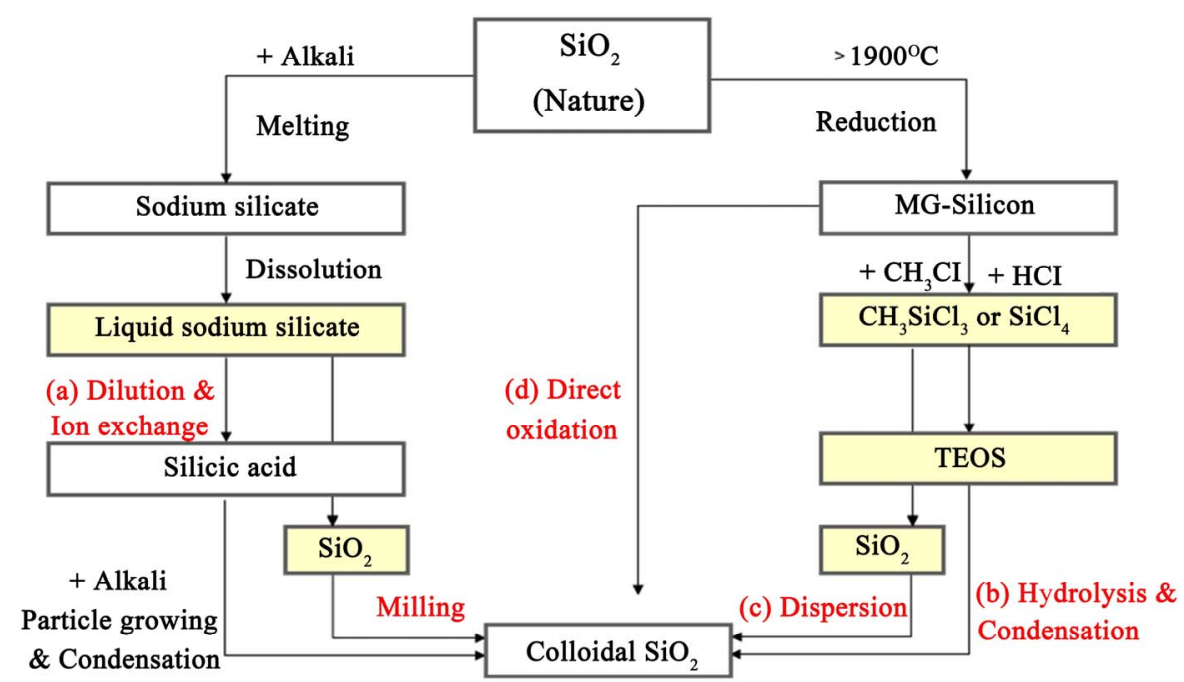

Figure 1. Various preparation methods for colloidal silica: (a) Ion exchange from sodium silicate; (b) Hydrolysis and condensation from TEOS; (c) Milling or dispersion from fumed silica; (d) Direct oxidation from silicon.

Table 1. Starting materials and preparation methods for colloidal silica.

\begin{tabular}{cc}
\hline Starting material & Preparation method \\
\hline \multirow{2}{*}{ Sodium silicate } & Ion exchange \\
& Neutralization \\
& Electrodialysis \\
\hline \multirow{2}{*}{ Silica gel } & Peptization \\
Silicon compound & Milling \\
\hline \multirow{2}{*}{ Silicon } & Hydrolysis \& Condensation \\
\hline
\end{tabular}

kali solution to form a silica seed, which is then used to grow silica particles. The product is concentrated to 30 wt $\%$ to obtain the commercial product. The second method for preparing colloidal silica is from tetraethoxysilane (TEOS), which is well known as the Stöber method [6]. TEOS is a silane monomer prepared from tetrachlorosilane, which in turn is derived from metallurgical-grade silicon. The silicon itself is obtained by the reduction of naturally occurring silica ore at temperature over $1900^{\circ} \mathrm{C}$ in the presence of carbon. The third method is that of direct oxidation of silicon wherein colloidal silica is prepared by the direct oxidation of metallurgical grade silicon without using TEOS. The silicon is treated with water in the presence of alkali catalysts to produce silica along with the evolution of hydrogen and heat. Finally, the fourth method for preparing colloidal silica is by milling and peptization of silica that can be found either in the form of silica gel or fumed silica, which consists of preferentially coaccervated or aggregated primary silica particles. The properties of the colloidal silica prepared by this route largely depend not only on the milling and peptization process but also on the prop- erties of the starting silica source such as purity, shape, and aggregation.

Colloidal silica is often defined by its solid content, counter ion type and concentration, $\mathrm{pH}$, and particle size of silica. However, when we used colloidal silica in various applications, we observed a few differences in the results, and therefore, we compared the characteri- stics of colloidal silica on the basis of the preparation method employed and starting materials used. A careful comparison of colloidal silica and the preparation meth- ods may help in choosing the method that is the most appropriate for the application being considered.

\section{Experimental}

\subsection{Colloidal Silica Prepared from Various Preparation Methods}

Seven colloidal silica samples having different particle sizes and preparation methods are listed in Table 2 . They were prepared by ion exchange of sodium silicate, hydrolysis and condensation of TEOS, milling and dispersion of fumed silica, and direct oxidation of silicon. The nominal size of their particles is 10,30 , and $60 \mathrm{~nm}$ and their concentration was adjusted to $10 \mathrm{wt} \%$ with $\mathrm{pH} \sim 10$ for the sake of comparison.

The colloidal silica prepared from sodium silicate by ion exchange has been described in detail in previous reports [3-5]. Samples with a nominal particle size of 10 $\mathrm{nm}$ and $60 \mathrm{~nm}$ (IE-10 and IE-60, respectively) were prepared by controlling the concentration, alkali source, and the particle growing step.

The properties of samples having a nominal particle 
Table 2. Comparison of typical samples prepared by various methods.

\begin{tabular}{|c|c|c|c|c|c|c|c|}
\hline \multirow{2}{*}{$\begin{array}{c}\text { Preparation process } \\
\text { Sample }\end{array}$} & \multicolumn{2}{|c|}{ Ion exchange } & \multicolumn{2}{|c|}{ Hydrolysis \& Condensation } & \multirow{2}{*}{$\begin{array}{c}\text { Milling \& Dispersion } \\
\text { MD-30 }\end{array}$} & \multicolumn{2}{|c|}{ Direct oxidation } \\
\hline & IE-10 & IE-60 & TE-10 & TE-60 & & DO-10 & DO-60 \\
\hline Counter ion & $\mathrm{Na}$ & $\mathrm{K}$ & $\mathrm{Na}$ & $\mathrm{K}$ & $\mathrm{K}$ & $\mathrm{Na}$ & $\mathrm{K}$ \\
\hline TEM size (nm) & 13 & 53 & 12 & 71 & 34 & 14 & 62 \\
\hline PSA size (nm) & 14 & 83 & 23 & 86 & 136 & 16 & 56 \\
\hline Aggregation factor ${ }^{*}$ & 1.0 & 1.6 & 1.9 & 1.2 & 4.0 & 1.1 & 0.9 \\
\hline $\mathrm{pH}$ & 9.2 & 9.7 & 10.5 & 10.3 & 10.7 & 9.6 & 10.1 \\
\hline
\end{tabular}

* PSA size/TEM size.

size of $10 \mathrm{~nm}$ and $60 \mathrm{~nm}$ (TE-10 and TE-60, respectively) obtained by the hydrolysis and condensation of TEOS were compared with those of other samples. The particle size of the silica prepared using TEOS can be controlled by choosing the solvent, catalyst, concentration of starting materials (TEOS, water, alcohol cosolvent, and alkali catalyst), temperature, reaction time, etc. The base fluid obtained after the synthesis of colloidal silica is a mixture of water and ethanol. Therefore, the base fluid is replaced with water through centrifugation three times at $10000 \mathrm{rpm}$ for $60 \mathrm{~min}$. Subsequently, its $\mathrm{pH}$ was adjusted to result in an alkaline solution.

The colloidal silica obtained from fumed silica was prepared by the dispersion of fumed silica (150 G, Tokuyama, Japan) using $\mathrm{KOH}$ such that the $\mathrm{pH}$ of the slurry was adjusted to $\sim 10$. The average silica particle size of the first dispersed phase obtained by means of ultrasonicfication is approximately $250 \mathrm{~nm}$; therefore, it was further milled in the presence of 0.5-mm-diameter zirconia beads using a sand mill (ALESCO) or nanofluidizer (M-110P, Microfluidics) to obtain an average particle size that is half the starting value, as measured by the dynamic light scattering method. The primary particle size obtained from fumed silica, as measured by a transmission electron microscope (TEM), is much smaller than the final average particle size obtained after milling, as measured by the light scattering method; therefore, the aggregated primary particles were not separated further. The primary particle size obtained from fumed silica was approximately $30 \mathrm{~nm}$, as measured by a TEM, and hence, the sample is called MD- 30 .

Colloidal silicas having two different average particle sizes, $10 \mathrm{~nm}$ and $60 \mathrm{~nm}$ (DO-10 and DO-60, respecttively) were prepared by the direct oxidation of silicon. The preparation method of direct oxidation has been described in detail in previous reports $[8,9]$.

\subsection{Characterization}

The primary particle size and shape of colloidal silica particles was examined by a TEM (2000EX, JEOL) at $200 \mathrm{kV}$. The $10 \mathrm{wt} \%$ sol was diluted to $0.5 \mathrm{wt} \%$ using distilled water along with $\mathrm{NaOH}$ so that the $\mathrm{pH}$ of the diluent was adjusted to 9.5. The diluted sol was subjected to ultrasonic treatment for 3 min and 2 drops of the diluted sample were dropped on a carbon coated 200-mesh grid over filter paper (5A, ADVANTEC) and dried at $60^{\circ} \mathrm{C}$ overnight for TEM observation.

The particle size analysis (PSA) was conducted by the dynamic light scattering method. The sample was diluted to $0.5 \mathrm{wt} \%$ to measure the zeta potential using a dynamic light scattering spectrometer (ELS-8000, Photal, Otsuka). The zeta potential curve, which shows the $\mathrm{pH}$ vs. the zeta potential, is obtained by preparing the colloidal silica and by varying its $\mathrm{pH}$ using $\mathrm{HNO}_{3}$ and $\mathrm{KOH}$.

The stability of the colloid is very important from the viewpoint of practical applications. It was measured by observing the aggregation or agglomeration at various $\mathrm{pH}$ conditions, and the turbidity was measured using Turbiscan (Turbiscan Lab, Formulation).

The cation concentrations of colloidal silica were determined by inductively coupled plasma optical emission spectroscopy (ICP-OES, OPTIMA 5300 DV, PerkinElmer). The cation concentrations of the base fluid were also checked by ICP-OES with the base fluids being separated out from the silica colloid by high-speed centrifugation.

The template is used to format your paper and style the text. All margins, column widths, line spaces, and text fonts are prescribed; please do not alter them. You may note peculiarities. For example, the head margin in this template measures proportionately more than is customary. This measurement and others are deliberate, using specifications that anticipate your paper as one part of the entire proceedings, and not as an independent document. Please do not revise any of the current designations.

\section{Results and Discussion}

The various colloidal silicas prepared by different methods from different starting materials are listed in Table 2. The average particle size and aggregation factor, measured using a TEM and by the dynamic light scattering method; type of counter ion; and $\mathrm{pH}$ are summarized. Various colloidal silicas having a primary particle size of approximately $10 \mathrm{~nm}$, and 60 70 $\mathrm{nm}$ were prepared by the ion-exchange of sodium silicate, direct oxidation of silicon, and hydrolysis and condensation of TEOS, whe- 
reas colloidal silicas having a primary particle size of 30 $\mathrm{nm}$ were prepared by the dispersion of fumed silica. For some samples, the average particle size measured by TEM image analysis matches the nominal size and the average particle size measured by the dynamic light scattering method is larger than the primary particle size measured by TEM. The aggregation factor is defined by the ratio of the PSA size to the TEM size, and it serves as an indication of the extent of the aggregation of primary particles to form secondary particles. In the case of silica sols having a nominal particle size of $10 \mathrm{~nm}$, the sample prepared by hydrolysis and condensation of TEOS (TE-10) has an aggregation factor that is higher than that of the samples prepared by other methods (IE-10 and DO-10), whereas for $60 \mathrm{~nm}$ silica sols, the sample prepared by the ion exchange of sodium silicate (IE-60) has a higher aggregation factor than that of the samples prepared by other methods (TE-60 and DO-60). The MD-30 sample has the highest aggregation factor (4.0) among all seven samples.

The colloids having a nominal particle size of $10 \mathrm{~nm}$ are generally more transparent than those having a size of $60 \mathrm{~nm}$. The smaller the size of the particle and the lesser the extent of aggregation, the higher was the transmittance. According to the transmittance plot for one day shown in Figure 2, all seven samples do not settle down or aggregate within the observation period. The transmittance of IE- 10 and DO-10 is the highest and that of TE- 10 is greater than that of the $30-\mathrm{nm}$ and $60-\mathrm{nm}$ colloids but lower than that of IE-10 and DO-10 because it has the highest aggregation factor among IE-10, DO-10, and TE-10. The transmittance of $60 \mathrm{~nm}$ colloids decreases in the order of DO-60, TE-60, and IE-60. The MD-30 sample has a primary particle size that is smaller than that of DO-60, TE-60, and IE-60; however, its transmittance is lower than that of DO-60 and TE-60 and greater than that of IE-60.

The zeta potential curves of 10-nm and 60-nm samples are generally similar to each other (-) in that for basic conditions (pH higher than 7), the zeta potential increases with a decrease in $\mathrm{pH}$ such that the isoelectric point (IEP) is located at a pH value of approximately 3 (Figure 3 ).

The zeta potential curves of DO-60, TE-60, and IE-60 are also similar to each other (-) in that for basic conditions, the zeta potential increases with a decrease in $\mathrm{pH}$. However, in this case, the IEP is slightly smaller than that for $10-\mathrm{nm}$ samples and the slope of the curve is slightly steeper between $\mathrm{pH} 2$ and 4 as compared to that for the 10-nm colloids; therefore, the $60-\mathrm{nm}$ samples have large absolute zeta potential values over a wider $\mathrm{pH}$ range of 4-10, whereas the $10-\mathrm{nm}$ samples have large absolute zeta potential values only in the $\mathrm{pH}$ range of $7-10$. This indicates that the stability of the silica sol

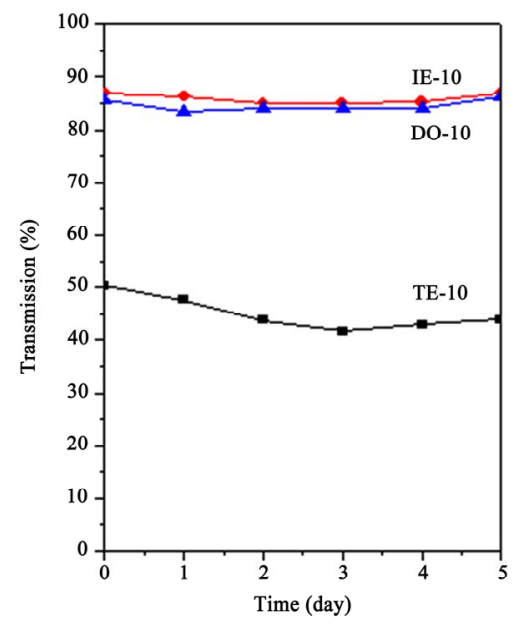

(a)

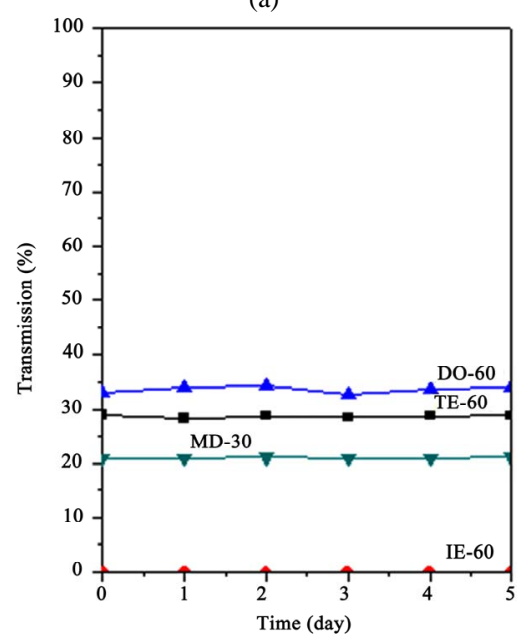

(b)

Figure 2. Comparison of change in transmittance with time for different samples of colloidal silica: (a) IE-10, TE-10, and DO-10; (b) IE-60, TE-60, DO-60, and MD-30.
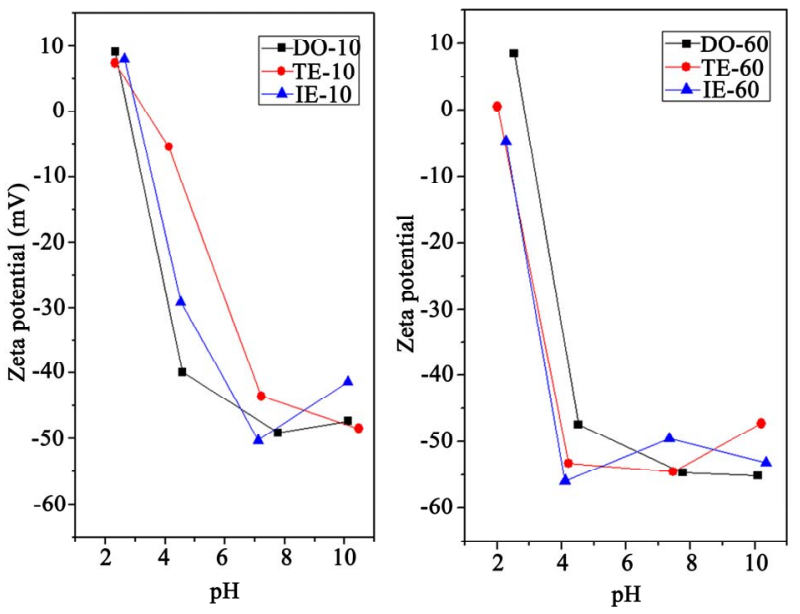

Figure 3. Zeta potential curve with $\mathrm{pH}$ variation for (a) samples having a nominal particle size of $10 \mathrm{~nm}$ and (b) samples having a nominal particle size of $60 \mathrm{~nm}$. 
having $60-\mathrm{nm}$ particles is better over a wider range of $\mathrm{pH}$, especially in the $\mathrm{pH}$ range of 4-7, as compared to that of the sol having $10-\mathrm{nm}$ particles. The zeta potential of the TE-10 sample at $\mathrm{pH} 4$ is relatively greater than that of IE-10 and DO-10 samples, which could be an indication of lower stability as a result of solvent substitution with water and the decrease in the number of cations in the Stern layer, which will be discussed later.

The stability of samples was compared with varying centrifugation speed and time. After centrifuge at 6,000 rpm for $1 \mathrm{hr}$, samples with $10 \mathrm{~nm}$ particles did not settle down but MD-30 sample revealed top 10 vol\%, DO-60 sample revealed top 30 vol\%, TE-60 and IE-60 samples revealed 90 vol\% transparent top. It depends on the size of the primary particles. The $10 \mathrm{~nm}$ samples were not settle down even after centrifuge at $10,000 \mathrm{rpm}$ for $1 \mathrm{~h}$ so that the difference in the stability of DO-10, TE-10, and IE-10 was not observed through centrifugation.

Figure 4 shows the TEM images of various silica sols, enabling a comparison of the silica particles in terms of their primary particle size and shape. The similar primary particles of the 60-nm samples (IE-60, TE-60, and DO-60) are compared. The IE-60 sample is aggregated as may be expected from its aggregation factor (1.9), whereas the TE-60 and DO-60 silica samples are mono-dispersed. Among the 60- and 10-nm colloids, the aggregation factor is largest for IE-60 and TE-10, respectively. The TEM image of the MD-30 sample is shown at low and high magnification in Figure 5, wherein the interconnecting bonds of the primary parti- cles are not fully separated even after milling for $1 \mathrm{~h}$. It should be noted that the cohesion between the particles originating from the spray pyrolysis step of the fumed silica preparation produced bridged primary particles, which are difficult to separate and dispersed completely to a mono-dispersed phase by milling and dispersion.

The cation concentrations of the colloidal silica are listed in Table 3. The 10-nm colloids have Na counterions whereas the 60 -nm ones have $\mathrm{K}$ counter-ions as the major stabilizing cations. The TE-10 and TE-60 samples have the lowest cation concentrations of $\mathrm{Na}$, however, DO-10 and DO-60 have cation concentrations of $\mathrm{K}$ that are lower than that of $\mathrm{Na}$ in $\mathrm{TE}-60$. The absolute value zeta potential value of the TE-10 sample at $\mathrm{pH} \sim 4$ was relatively larger than that of the other two 10-nm samples. This could be because of the small number of cations on the surface of silica particles, which determines the stability of the colloid. This layer might be a Stern layer. The $\mathrm{Na}$ concentration is similar for both the ion-exchanged samples and the direct-oxidation samples.

It is very interesting to note the concentration of $\mathrm{Na}$ in the base fluids, as shown in Table 4. The ion concentration after the separation of silica particles is considerably low in the IE and TE samples, however, the ion concentration in the DO-10 and DO-60 samples is not as low as that in other samples. The cations present in the base fluid are known as free cations, those on the surface of silica, as bound cations; and those in the silica, as incurporated cations. By comparing the cation concentrations in the base fluid (Table 4) and the cation concentrations in the silica colloid, it is found that the difference between the cation concentrations in the base fluid and in the silica colloid is larger in IE and TE samples than DO samples. It can be characterized by the fraction of free cations, which is the ratio of cation concentration in the fluid divided by cation concentration in the particle (Table 4 and Figure 6). The cation concentration is lower in the TE-10 sample but the fraction of free cations is the
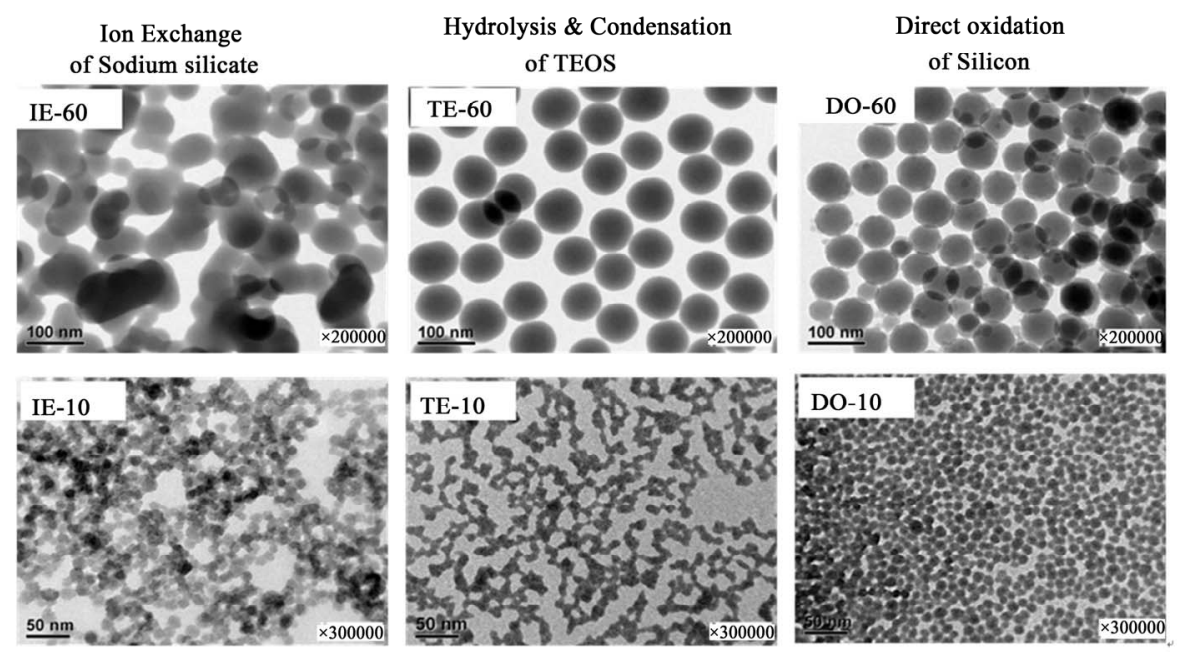

Figure 4. Comparison of TEM morphologies of colloidal silica in IE-60, IE-10, TE-60, TE-10, DO-60, and DO-10. 


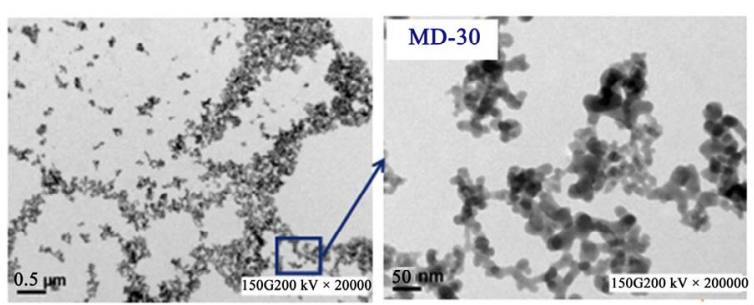

Figure 5. TEM morphology of colloidal silica in MD-30.

Table 3. ICP analysis of cations in colloidal silica (mg/kg).

\begin{tabular}{ccccccc}
\hline Cation & IE-10 & IE-60 & TE-10 & TE-60 & DO-10 & DO-60 \\
\hline $\mathrm{Na}$ & 911 & 81.7 & 189 & 22.4 & 988 & 89.4 \\
$\mathrm{~K}$ & 5.1 & 3468 & 21.5 & 2011 & 6.7 & 1322 \\
$\mathrm{Ca}$ & 21.6 & 1.8 & - & - & 2.4 & 3.8 \\
$\mathrm{Fe}$ & 8.7 & 0.8 & 3.0 & 0.3 & 2.1 & 0.8 \\
\hline
\end{tabular}

Table 4. ICP analysis of cations in base fluid of colloidal silica and fraction of cations in base fluid.

\begin{tabular}{|c|c|c|c|c|c|c|c|}
\hline \multicolumn{2}{|c|}{ Cation (mg/kg) } & \multirow{2}{*}{$\frac{\text { IE-10 }}{102}$} & \multirow{2}{*}{$\begin{array}{c}\text { IE-60 } \\
6.6\end{array}$} & \multirow{2}{*}{$\begin{array}{c}\text { TE-10 } \\
34\end{array}$} & \multirow{2}{*}{$\begin{array}{c}\text { TE-60 } \\
5.2\end{array}$} & \multirow{2}{*}{$\begin{array}{r}\text { DO-10 } \\
323\end{array}$} & \multirow{2}{*}{$\frac{\text { DO-60 }}{24}$} \\
\hline Cation & $\mathrm{Na}$ & & & & & & \\
\hline $\begin{array}{c}\text { concentration } \\
\text { (mg/Kg) }\end{array}$ & K & 0.58 & 360 & - & 289 & 1.8 & 231 \\
\hline $\begin{array}{l}\text { Fraction of } \\
\text { free cations } \\
\text { (cations in } \\
\text { base fluid/ }\end{array}$ & $\mathrm{Na}$ & 0.12 & 0.09 & 0.22 & 0.30 & 0.49 & 0.37 \\
\hline $\begin{array}{l}\text { cations in } \\
\text { particle) }\end{array}$ & $\mathrm{K}$ & 0.12 & 0.11 & 0 & 0.16 & 0.37 & 0.21 \\
\hline
\end{tabular}

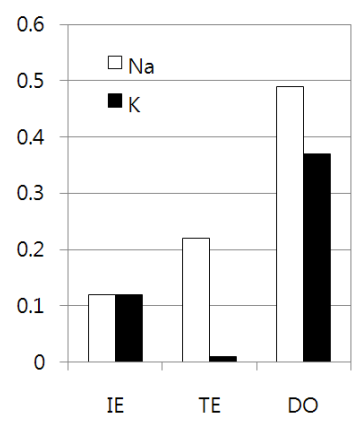

(a)

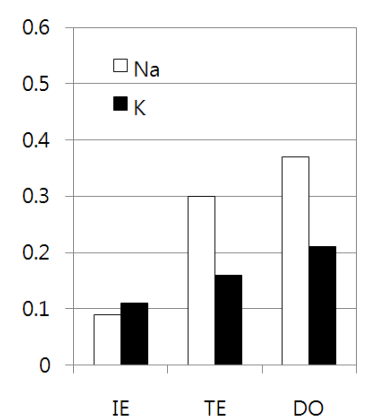

(b)
Figure 6. Comparison of fraction of free cation in (a) $\mathbf{1 0} \mathbf{~ n m}$ samples and (b) $60 \mathrm{~nm}$ samples.

lowest in the IE-10 sample. This allows us to postulate that the fraction of free cations, i.e., the proportion of the total number of cations present in the particle, differs depending on the preparation methods. The fraction of free cations in DO samples is larger than that in IE and TE samples. In other words, the number of cations located in the silica particles in DO samples is lower than that in the IE and TE samples; therefore, the elimination of sodium cations is relatively easier in the DO-10 and
DO-60 samples and can be carried out by an additional washing step such as centrifugation or ultra membrane filtration to produce silica sol with lower cation concentration.

Although the schematic plot shown in Figure 7 attempts to classify the samples based on the number of $\mathrm{Na}$ cations present in the particle and the fluid, the cations could either be present inside the particle or be exposed on the surface. However, the fractions of cation in the fluid of $10 \mathrm{~nm}$ samples are overall higher than that of 60 $\mathrm{nm}$ samples, which could imply that the cation is incorporated inside the particle rather than the surface. If it is located on the surface of silica, the higher amount of $\mathrm{Na}$ would be attached on the surface of silica due to higher surface area, so that the lower fraction of $\mathrm{Na}$ is expected in $10 \mathrm{~nm}$ samples. The disparity in the location of the cations can be well understood by considering the reaction mechanism of each preparation method. The IE samples were prepared from sodium silicates, and hence, the incorporation of sodium ions in the particle is unavoidable; therefore it is difficult to completely eliminate the cations by ion exchange in the extreme limit, such that the concentration of cations is lower than $10 \mathrm{ppm}$ at $10 \mathrm{wt} \%$ concentration of solid contents in the silica colloid. However, the DO samples were prepared from silicon in the presence of an alkali catalyst, where the alkali molecule helps to dissolve the silicon molecule to form a hydrolyzed silicon monomer, which undergoes condensation to form a Si-O-Si bridge with another hydrolyzed silicon monomer; therefore, the alkali catalyst acts as a carrier and helps in carrying silicon molecules one by one from the silicon powder to form a silica.

The four different methods for preparing colloidal silica are compared in Table 5 on the basis of starting materials, main reaction, extent of the aggregation of resulting particles, cation concentrations, and economic considerations. The advantages and drawbacks of each preparation method are listed. The disadvantage of the ion exchange method is the presence of residual $\mathrm{Na}$ in the low limit and the environmental costs of recovering the ion exchange resin. The disadvantage of the hydrolysis and condensation method are the costs of recovering the solvents and their relatively high price, even though this method produces high purity silica and provides good control over the particle morphology. Finally, the direct oxidation method provides monodispersed particles at reasonable costs and purity control; however, the filtering step needs to be carried out carefully to separate silicon sludge from silica particles after direct oxidation. Each method has its own advantages and disadvantages, and therefore, the method chosen depends on the type of colloidal silica required for a particular application field. The colloidal silica has unique characteristics in the pu- 
Ion exchange

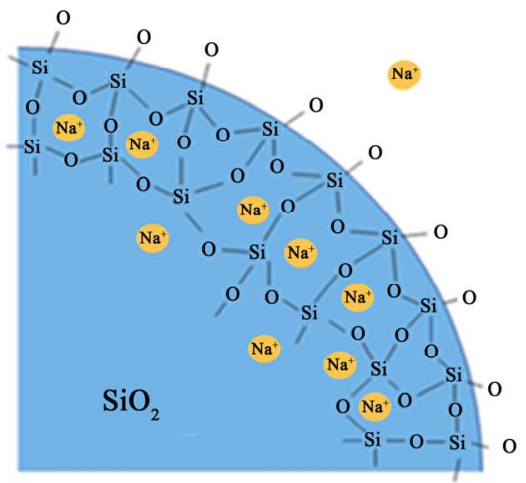

Hydrolysis \& condensation

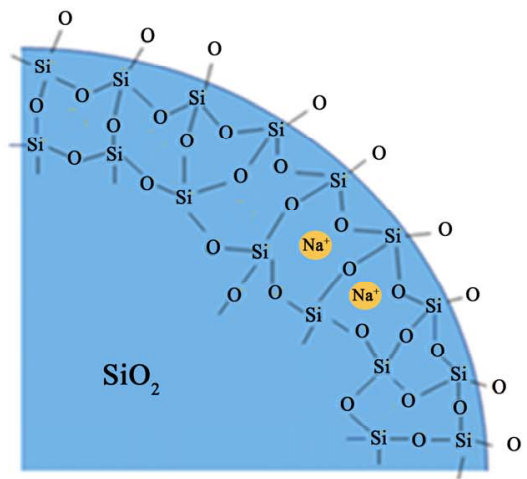

Direct oxidation

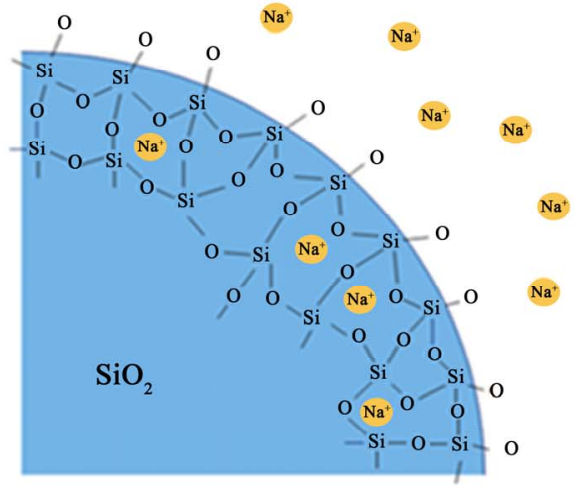

Figure 7. Comparison of the schematic presentation of cation locations in colloidal silica prepared (a) by ion exchange of sodium silicate and (b) by direct oxidation of silicon.

Table 5. Comparison of preparation methods of silica sol.

\begin{tabular}{|c|c|c|c|c|}
\hline Class & Ion exchange & $\begin{array}{c}\text { Hydrolysis \& Condensa- } \\
\text { tion }\end{array}$ & Dispersion & Direct oxidation \\
\hline $\begin{array}{l}\text { Starting Materi- } \\
\text { al }\end{array}$ & Sodium silicate & $\mathrm{Si}\left(\mathrm{OCH}_{2} \mathrm{CH}_{3}\right)_{4}$ & Silica & Silicon \\
\hline Main Reaction & $\begin{array}{l}\text { Dilution } \\
\text { Ion exchange } \\
\text { Growing } \\
\text { Concentration }\end{array}$ & $\begin{array}{l}\text { Hydrolysis \& } \\
\text { Condensation } \\
\text { Concentration }\end{array}$ & $\begin{array}{l}\text { Milling or dispersion } \\
\text { concentration }\end{array}$ & $\begin{array}{l}\text { Oxidation } \\
\text { Filter }\end{array}$ \\
\hline Aggregation & $\begin{array}{l}\text { Some aggrega- } \\
\text { tion }\end{array}$ & Mono disperse & Aggregation & Mono disperse \\
\hline Imurity & High & Lowest & Low & Low \\
\hline Price & Reasonable & High & Reasonable & Reasonable \\
\hline Advantage & Easy size control & $\begin{array}{l}\text { Monodisperse } \\
\text { High purity }\end{array}$ & $\begin{array}{c}\text { High purity } \\
\text { One step for organic solvent based } \\
\text { fluid }\end{array}$ & $\begin{array}{c}\text { Reduced waste water } \\
\text { No need for concentration } \\
\text { Control of } \mathrm{Na}\end{array}$ \\
\hline Disadvantage & $\begin{array}{l}\text { Residual Na } \\
\text { Recycle of ion } \\
\text { exchange resin }\end{array}$ & $\begin{array}{l}\text { Recycle of solvent } \\
\text { High price }\end{array}$ & Size \& shape limitation & Filter process \\
\hline
\end{tabular}

rity and shape control depending on the preparation methods, so that it might be useful for whom uses silica sol in various application.

\section{Acknowledgements}

This study was supported by the Energy Resources Technology R\&D program (R200811049) under the Ministry of Knowledge Economy, Republic of Korea.

\section{References}

[1] C. C. Payne, "Applications of Colloidal Silica: Past, Present, and Future,” In: H. E. Bergna, Ed., The Colloid Chemistry of Silica, Advances in Chemistry, Series 234, Chapter 29, American Chemical Society, Washington DC, 1994, pp. 581-594.

[2] R. K. Iler, “The Chemistry of Silica,” Wiley, New York, 1979, pp. 331-337.
[3] A. Yoshida, "Silica Nucleation, Polymerization, and Growth Preparation of Monodispersed Sols,” In: H. E. Bergna, Ed., The Colloid Chemistry of Silica, Advances in Chemistry, Series 234, Chapter 2, American Chemical Society, Washington DC, 1994, pp. 51-62.

[4] M.-S. Tsai, P. Y. Huang and W.-C. Wu, "The Study of Formation Process of Colloidal Silica from Sodium Silicate," Materials Research Bulletin, Vol. 40, No. 9, 2005, pp. 1609-1616.

[5] M.-S. Tsai, “The Study of Formation Colloidal Silica via Sodium Silicate,” Materials Science and Engineering: B, Vol. 106, No. 1, 2004, pp. 52-55.

[6] W. Stöber, A. Fink and E. Bohn, "Controlled Growth of Monodisperse Silica Spheres in the Micron Size Range," Journal of Colloid and Interface Science, Vol. 26, No. 1, 1968, pp. 62-69.

[7] Y.-K. Lee, Y. R. Yoon and H.-K. Rhee, "Preparation of Colloidal Silica Using Peptization Method,” Colloids and Surfaces A: Physicochemical and Engineering Aspects, Vol. 173, No. 1, 2000, pp. 109-116. 
[8] H. M. Lim, H. C. Shin, S. H. Huh and S. H. Lee, "Effect of Catalyst on the Colloidal Silica Particle Growth in Direct Oxidation of Silicon," Solid State Phenomena, Vol. 124-126, 2007, pp. 667-670.

[9] W. K. Na, H. M. Lim, S. H. Huh, S. E. Park, Y.-S. Lee and S. H. Lee, "Effect of the Average Particle Size and the Surface Oxidation Layer of Silicon on the Colloidal
Silcia Particle through Direct Oxidation,” Materials Science and Engineering: B, Vol. 163, No. 2, 2009, pp. 82-87.

[10] J. J. Guo, X. H. Liu, Y. C. Cheng, Y. Li, G. J. Xu and P. Cui, "Size Controllable Synthesis of Monodispersed Colloidal Silica Nanoparticles via Hydrolysis of Elemental Silicon,” Journal of Colloid and Interface Science, Vol. 326, No. 1, 2008, pp. 138-142. 